\title{
Contribuição à manutenção da via permanente com acelerômetros em pacotes inerciais portáteis
}

\author{
Contribution to the maintenance of the permanent track with accelerometers in portable inertia
} packages

Contribución al mantenimiento de la vía permanente con acelerómetros en paquetes inerciales portátiles

\author{
João Bosco de Lima \\ ORCID: https://orcid.org/ 0000-0002-5723-0523 \\ Instituto Militar de Engenharia, Brasil \\ E-mail: engferjbosco@gmail.com \\ Luis Antônio Silveira Lopes \\ ORCID: https://orcid.org/0000-0001-9325-4330 \\ Instituto Militar de Engenharia, Brasil \\ E-mail: laslopes@ime.eb.br \\ Luiz Augusto Cavalcante Moniz de Aragão Filho \\ ORCID: https://orcid.org/0000-0001-6676-1396 \\ Instituto Militar de Engenharia, Brasil \\ E-mail: moniz@ime.eb.br
}

\begin{abstract}
Resumo
O monitoramento de condições dos sistemas ferroviários tem sido amplamente utilizado para apoiar a avaliação da integridade de suas estruturas. A inspeção geométrica da via permanente é um dos principais métodos utilizados para a elaboração de planos de manutenção da malha ferroviária. Sabe-se que as intervenções são baseadas nas indicações de inconformidades, de acordo com intervalos pressupostos para os parâmetros analisados. Entretanto, mesmo com a referida inspeção, continuam a ocorrer descarrilamentos oriundos da superestrutura. $\mathrm{O}$ artigo apresenta a utilização de um dispositivo inercial em seis trechos ferroviários, de forma a complementar as inspeções geométricas e priorizar a escala de manutenção. A execução deste estudo justifica-se, pois, os equipamentos comumente utilizados na medição da dinâmica de via possuem preços elevados e alta complexidade de instalação. $\mathrm{O}$ acelerômetro empregado é de baixo custo e apresenta fácil aplicação. O referido dispositivo foi capaz de identificar pontos críticos de interação veículo-via, que não apresentavam exceção de geometria.
\end{abstract}

Palavras-chave: Superestrutura ferroviária; Descarrilamento; Acelerômetro.

\begin{abstract}
Monitoring of the condition of railway systems has been widely used to support the assessment of the integrity of its structures and infrastructures. Permanent way geometric inspection is one of the main methods used for maintenance plans elaboration in railway networks. It is known that interventions are based on indications of nonconformities, according to intervals assumption for the analyzed parameters. However, even with this inspection, derailments originated in superstructure continues to occur. This paper presents the use of an inertial device in six railway sections, to complement the geometric inspections and prioritize maintenance scale. The execution of this study is justified because the equipment commonly used in track dynamics measurement have high prices and high complexity of installation. The accelerometer used is inexpensive and easy to apply. The mentioned device was able to identify critical points of vehicle-path interaction, that did not present geometry exception.
\end{abstract}

Keywords: Railway superstructure; Derailment; Accelerometer.

\section{Resumen}

La supervisión del estado de los sistemas ferroviarios se ha utilizado ampliamente para respaldar la evaluación de la integridad de sus estructuras e infraestructuras. La inspección geométrica de la vía permanente es uno de los principales métodos utilizados para la elaboración de planes de mantenimiento de la red ferroviaria. Se sabe que las intervenciones se basan en indicaciones de inconformidades, según los intervalos asumidos para los parámetros analizados. Sin embargo, incluso con esta inspección, siguen ocurriendo descarrilamientos de la superestructura. El artículo presenta el uso de un dispositivo inercial en seis tramos ferroviarios, con el fin de complementar las inspecciones geométricas y priorizar la escala de mantenimiento. La ejecución de este estudio está justificada, por lo tanto, el equipo comúnmente 
utilizado en la medición de la dinámica de la pista tiene precios altos y alta complejidad de instalación. El acelerómetro empleado es de bajo costo y cuenta con una aplicación fácil. Este dispositivo fue capaz de identificar puntos críticos de la interacción vehículo-pista, que no tenía ninguna excepción de geometría.

Palabras clave: Superestructura ferroviária; Descarrilamiento; Acelerómetro.

\section{Introduçãa}

Os custos envolvidos na implantação de um sistema ferroviário são muito elevados, destacando-se os gastos com a construção da via permanente e a compra dos veículos (Pastori, 2007). Por isso, deseja-se que o vultoso capital investido tenha o retorno planejado, mitigar ou eliminar eventos que causem problemas ao sistema é importante ação para evitar prejuízos ao investimento (Valinho et al., 2020). A manutenção dos elementos da superestrutura (trilhos, dormentes, fixações, lastro e sublastro) e da infraestrutura (subleito, dispositivos de drenagem e obras de arte especiais) torna-se então primordial e deve ser gerida de acordo os critérios estabelecidos em normas ou diretrizes para que a via apresente condições ao adequado rolamento das composições.

Apesar de todo o avanço tecnológico para a garantia do nível de serviço, as ferrovias têm apresentado um número considerável de falhas e acidentes que acabam por impactar de forma negativa a qualidade do transporte e gerar despesas adicionais às empresas (Ngamkhanong et al., 2018). Nesse âmbito, os descarrilamentos destacam-se como um problema, que gera muitos prejuízos (Liu et al., 2011). Tal fenômeno pode acarretar interrupções do tráfego, perdas da carga transportada, diminuição da vida útil dos ativos, impactos ao meio ambiente, necessidade de obras de reparo e até mesmo ferimentos graves com vítimas fatais.

Para que a ocorrência de descarrilamentos seja reduzida, é ideal que se realize manutenções preventivas e corretivas da superestrutura ferroviária (Kasraei \& Zakeri, 2020). No Brasil, no entanto, tais manutenções são comumente baseadas em parâmetros obtidos das inspeções geométricas da via permanente (Ratton Neto, 1985) e ainda, raramente, através de inspeções dinâmicas com equipamentos de alto custo e difícil operação, como por exemplo, a instrumentação de truques. A inspeção de geometria realizada com veículos tipo carros controle (ex.: TrackSTAR, EM80 Plasser e Ensco Car) disponibilizam adequadas informações da via através da medição de vários parâmetros importantes, entretanto quando analisados de forma separada, não são capazes de avaliar como as composições em operação interagem com a via. Já a inspeção dinâmica possibilita fazer uma análise mais elaborada da região, no tocante às excitações verticais e horizontais oriundas das imperfeições da geometria que prejudicam a interação veículo-via.

A pesquisa e a análise de dados realizadas buscam divulgar a importância das acelerações verticais e horizontais obtidas na interação veículo-via. Saber onde essas acelerações acontecem juntamente com exceções de geometria são informações que se bem trabalhadas nos planos de manutenção através de ações corretivas mitigam os riscos de descarrilamentos, evitando perda de recursos com reparação de ativos danificados, além do impacto na imagem da operadora, que as vezes mesmo realizando trabalhos árduos e bem-intencionados se deparam com essas não conformidades por ignorar ou não perceber a complementariedade entre dinâmica e geometria (Kasraei \& Zakeri, 2020).

Nesse contexto, o objetivo deste estudo é demonstrar a grande valia de se utilizar inspeções dinâmicas por vibração, com ferramentas inerciais portáteis e de simples instalação, para complementar as inspeções geométricas e, desta forma, possibilitar a definição de planos de manutenção mais assertivos. Busca-se dar notoriedade à importância da análise das acelerações verticais e horizontais obtidas na interação veículo-via e como consequência minorar os descarrilamentos nas vias férreas. Agentes causadores de acelerações indesejadas podem ser causa principal ou contribuidora de descarrilamentos. 


\section{Revisão Bibliográfica}

\subsection{A Via Permanente: Geometria e Dinâmica Ferroviária}

A via permanente possui como principal função fornecer uma superfície resistente, com poucas irregularidades e capacidade de distribuir as cargas muito elevadas provenientes das passagens dos trens às camadas geotécnicas que a constituem, propiciando assim uma boa interação veículo/via. Em geral, ela é dividida em três partes principais: (i) a superestrutura, também conhecida como grade ferroviária, que é composta de trilhos, dormentes, itens de fixação e aparelhos de mudança de via; (ii) o pavimento ferroviário, composto de lastro, sublastro e subleito; (iii) elementos de drenagem, pontes, túneis, passagens de nível e obras de contenção pertencentes a região de circulação dos trens (Selig \& Waters, 1994).

Cada um dos elementos supramencionados possui sua missão dentro da via permanente. Os trilhos apoiam e orientam as rodas dos trens. Os dormentes e os itens de fixação distribuem as cargas aplicadas nos trilhos e os mantém com espaçamento constante. Os aparelhos de mudança de via direcionam os trens às transferências de linha permitindo cruzamento entre trens. $\mathrm{O}$ lastro garante o amortecimento da maioria das vibrações provocadas pelo rolamento dos trens, distribui as cargas e drena a água superficial. O sublastro absorve parte das cargas provenientes do tráfego de trens e as transmite ao subleito, que funciona como a camada de fundação da estrutura. Os elementos da infraestrutura possibilitam a drenagem da água da plataforma ferroviária e garantem que o trajeto projetado seja realizado pelos trens (Campos, 2019; Selig and Waters, 1994).

Conhecida a estrutura das linhas férreas, foram concebidos ao longo dos anos de operação elementos ou parâmetros de controle para garantir a adequada circulação dos trens. Nesse sentido, as primeiras avaliações da condição de uma ferrovia baseavam-se principalmente na chamada geometria de via, que engloba como parâmetros principais: bitola, superelevação, nivelamento longitudinal e alinhamento.

A bitola é a distância entre os trilhos da via medida entre as faces internas dos boletos e a característica fundamental do traçado, que também determina as características dos veículos. Tal parâmetro pode sofrer uma variação repentina, e, nesse momento, a distância entre os trilhos vir a ser maior ou menor que o eixo de apoio das rodas. Dessa forma, os trens podem descarrilar e cair dentro da via ou sofrerem desgaste excessivo de suas rodas cônicas devido ao contato do friso da roda com os trilhos (Brina, 1983).

A superelevação é a diferença de cota entre o topo dos trilhos, obtida pela inclinação da via, por meio da elevação de altura entre as filas de trilhos constituintes da grade. A maior altura do trilho externo em relação ao interno é a que se determina de superelevação. O referido parâmetro é utilizado para combater o resultado da ação da força centrífuga no sentido do trilho externo que age sobre o veículo em curva. Quando ocorre superelevação, o atrito nos frisos das rodas é aumentado e pode causar o tombamento do veículo. Para minimizar o efeito nocivo desta força, a via é inclinada em um determinado ângulo de elevação, gerando uma diferença de altura entre trilhos para criar uma componente de equilíbrio (Brina, 1983).

O empeno é um desnivelamento transversal, dado pela diferença de superelevação medida entre dois pontos e a distância entre esses dois pontos é chamada de base do empeno. O conceito de flecha nos ajuda na definição do nivelamento longitudinal e alinhamento, pois utilizando-se uma linha imaginária ou física que une dois pontos coleta-se a distância entre a linha e o trilho no ponto médio desta linha também denominada corda, esta distância é a flecha. Para medir as flechas e consequentemente definir nivelamento e alinhamento podemos usar vários tamanhos de corda, porém os tamanhos mais comuns são $10 \mathrm{~m}$ e $20 \mathrm{~m}$. Observa-se que tanto no nivelamento, quanto no alinhamento, quanto maior a flecha maior o parâmetro medido, segue outra maneira de conceituar uma flecha, é a distância perpendicular entre a linha de bitola no boleto dos trilhos e o centro de uma corda entre dois pontos desse mesmo trilho, sendo a medida que define a curvatura em planta da via.

Conforme cita Iwnick (2006), a dinâmica de uma ferrovia apresenta alta complexidade de análise. As composições ferroviárias podem possuir grande quantidade de veículos que interagem entre si e com a via permanente. Somadas as variáveis 
do modo de operação (condução), o comportamento do sistema torna-se bem complexo. Nesse sentido, a Dinâmica de Sistemas Multicorpos surge como uma alternativa para tentar explicar a dinâmica ferroviária. Esta teoria está baseada no desenvolvimento e solução de equações não-lineares, resolvidas com o auxílio de programas computacionais e nos conceitos de vibração.

A Tabela 1 correlaciona os movimentos dos veículos com parâmetros geométricos de via permanente de maior correlação e demonstra como os parâmetros de nivelamento e alinhamento influenciam na interação veículo-via e quando a geometria da via possui não conformidades destes parâmetros qual o comportamento da caixa dos veículos ao circular sobre esta região. Nota-se que pode acontecer a concomitância, o que aumenta a intensidade do movimento e os tipos de movimentos, mas todos serão registrados na inspeção de dinâmica que utilizam pacotes inerciais.

Tabela 1. Descrição dos movimentos dos veículos e do parâmetro condicionante da avaliação da geometria da via.

\begin{tabular}{|c|c|c|}
\hline Movimento & Descrição & Parâmetro Condicionante \\
\hline Pitch & Ora uma extremidade da caixa do veículo é sobrecarregada e a outra aliviada. & Nivelamento Longitudinal \\
\hline Bounce (Galope) & $\begin{array}{l}\text { As molas dos truques dianteiro e traseiro são comprimidas ao mesmo tempo } \\
\text { ocasionando trepidação e sobrecarrega em todos os eixos. }\end{array}$ & Nivelamento Longitudinal \\
\hline Yaw & $\begin{array}{l}\text { As extremidades da caixa se locomovem opostas transversalmente e causam um } \\
\text { desgaste maior das rodas pois há um choque no contato destas com o trilho. }\end{array}$ & Alinhamento \\
\hline Sway & Gera-se um desconforto causado por uma movimentação lateral do veículo. & Alinhamento \\
\hline Roll (Balanço) & $\begin{array}{l}\text { Movimento no sentido perpendicular à via, sobrecarregando ora as rodas de um lado do } \\
\text { veículo, ora as rodas do outro lado. }\end{array}$ & Nivelamento Longitudinal \\
\hline
\end{tabular}

Fonte: Iwnick (2006).

A análise dinâmica da via permanente inicia-se pela avaliação da geometria da linha, termo que se refere à posição que cada fila de trilho ocupa no espaço. Mesmo em uma via perfeita, a posição das filas varia no sentido longitudinal por meio de sucessivas inclinações e concordâncias verticais, formando o perfil altimétrico da via. Da mesma forma, a posição das filas no sentido transversal está sujeita às seções de tangentes ou retas interligadas por trechos curvos, formando o perfil planimétrico da via. Outro parâmetro importante a ser analisado na dinâmica ferroviária é a flexibilidade da linha, que se denota pelas características dos vagões, carregados ou não, que circulam pela via permanente (Lopes \& Ciriaco, 2017), ou seja, a via permanente possui um módulo de elasticidade inerente à superestrutura de projeto e a elasticidade apesar de ser inerente ao projeto gera movimentos nos veículos, porém movimentos que alteram muito pouco a boa interação veículo-via.

Os descarrilamentos são acidentes ferroviários em que os veículos deixam de apoiar um ou mais conjuntos de truque e rodas nos trilhos. A ocorrência destes fenômenos pode ser analisada de forma probabilística, como decorrência de fatores (defeitos) do material rodante, da superestrutura e falhas humanas, fazendo os trens deixarem a superfície de rolamento do trilho e tocar o chão (Indian Railways Institute of Civil Engineering, 2014).

\subsection{Dinâmica dos Descarrilamentos}

Os mecanismos de ocorrência de um descarrilamento têm sido estudados por diversos autores. Cherchas (1981) investigou as probabilidades de escalada de roda variando o ângulo de contato e o coeficiente de atrito. Zeng e Wu (2008) demonstram que há casos em que a equação de Nadal pode ser mais conservadora que métodos analíticos. Liu e Magel (2012) revisaram os elementos básicos e fatores associados aos descarrilamentos. Vlakhova (2015) abordou o descarrilamento sob a ótica de equações diferenciais, aplicando conceitos matemáticos de mecânica dos sólidos.

O modelo obtido permitiu estimar analiticamente as condições de descarrilamento, que dependem da forma do flange, raio de curvatura da via, altura do centro de massa do veículo, forças de interação veículo-via, as forças e momentos de perturbação externos e coeficientes de atrito das superfícies que se interagem. Simulações computacionais sobre 
descarrilamentos também vem sendo abordadas. Morales-Ivorra (2016) verificaram o efeito da falha dos aparelhos de fixação sobre as forças dinâmicas produzidas no contato roda-trilho utilizando o software Vampire. Wang e Li (2012) desenvolveram um modelo em MATLAB baseado na solução exata do ponto e da força de contato roda/trilho. Arivazhagan et al. (2015) trabalharam na elaboração de um sistema de inspeção automático de descarrilamento a partir de análise de imagens de escalada de rodas conjugadas com um algoritmo. Os resultados das simulações revelaram que as acelerações desempenham um importante papel no comportamento dinâmico do sistema, influenciando fortemente o risco de descarrilamento. Outros autores têm se

esforçado para realizar medições com uso de acelerômetros para auxiliar na análise e prevenção dos descarrilamentos, como é o caso deste estudo. Boogaard et al. (2018) apresentam uma configuração que mede a aceleração e as forças em um aparelho de mudança de via durante a passagem de trens. A partir das acelerações, tanto o deslocamento quanto o contato roda-trilho puderam ser analisados. Salvador et al. (2016) realizaram testes na Rede Ferroviária Metropolitana de Valência (Espanha) e as acelerações obtidas no truque determinaram as frequências ótimas de amostragem, bem como a localização dos acelerômetros ao longo do trem. Diversos defeitos de trilho, singularidades de trajetória e modos de vibração puderam ser claramente identificados. Real et al. (2012) desenvolveram um sistema que registra acelerações verticais sofridas pelos truques dos veículos ferroviários em circulação e apresentaram um procedimento de coleta de dados, o processamento matemático das acelerações e análise das condições da via.

\subsection{Descarrilamentos e Técnicas de Inspeção da Via Permanente}

Para o entendimento dos fatores que podem ocasionar descarrilamentos, é importante conhecer conceitos relativos à interação do veículo com a via. O critério proposto, baseado na mecânica estática, leva em consideração as forças laterais envolvidas no sistema roda/trilho, que não devem ser próximas ou superiores às forças verticais. Caso contrário, a probabilidade de ocorrer o descarrilamento é grande, conforme explicita a Equação (1).

$$
\frac{\mathrm{L}}{\mathrm{V}}=\frac{\tan \mathrm{B}-\mu}{1+\mu \tan \mathrm{B}}
$$

Em que:

L é a carga na direção horizontal no friso da roda (kN ou kgf);

V é a carga vertical atuante na roda ( $\mathrm{kN}$ ou kgf);

$B$ é o ângulo do ponto de contato entre a roda e o trilho;

$\mu$ é o coeficiente de atrito entre o lado interno do trilho e o friso da roda.

Na Figura 1 são ilustradas as componentes da Equação (1) (carga na direção horizontal L, e vertical V, ângulo de contato $B$ e o coeficiente de atrito $\mu$ ) no ponto de contato entre a roda e o trilho ferroviário (P) de aplicação do carregamento (N). 
Figura 1. Contato Roda-Trilho L/V

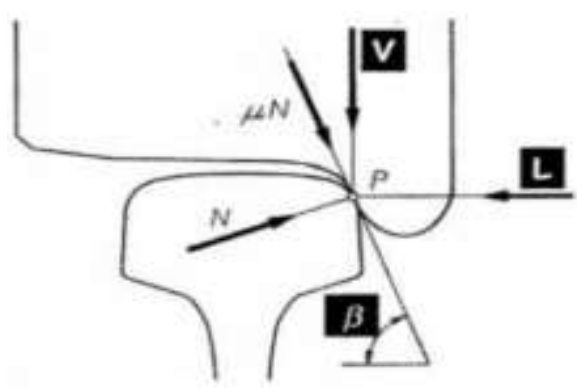

Fonte: Vlakhova (2015).

Com base em um coeficiente de atrito $\mu$ de 0,5 e um ângulo de flange $\beta$ de 67 graus, um limiar de L/V de 0,8 que é comumente adotado em estudos de segurança ferroviária (Liu \& Magel, 2012), entretanto, conforme cita Vlakhova (2015), nem sempre a equação de Nadal garante a não ocorrência de um descarrilamento. Ainda, segundo Santos \& Barbosa (2016), os critérios para análise de descarrilamentos, majoritariamente, não consideram a dinâmica do veículo e a geometria da via permanente em conjunto.

As acelerações horizontais e verticais são inerentes ao rolamento do material rodante sobre a via permanente, denominada interação veículo/via, se esta interação é adequada as acelerações ficam dentro de limites que não afetam significativamente a relação $\mathrm{L} / \mathrm{V}$, porém quando esta interação não é adequada geram acelerações significativas que torna a relação L/V igual ou acima do limite crítico de 0,8 comumente utilizado para se realizar uma avaliação funcional das ferrovias, sob a ótica da segurança da carga, passageiro e conforto do passageiro, como por exemplo o limite estabelecido na norma europeia (DIN EN 12229, 2009). Como existe uma correlação entre as acelerações verticais e horizontais das composições, com as forças verticais e horizontais atuantes no contato roda/trilho, dada pelos parâmetros de aceleração vertical e horizontal, então podemos expressar tal relação pela Equação (2) (Sato \& Umehara, 1987).

$$
\mathrm{ah}=-3 \mathrm{~L} \cdot 8 \mathrm{~V} \cdot \mathrm{av}+\mathrm{L} \cdot 2 \mathrm{~V}
$$

Em que:

ah é a aceleração horizontal;

av é a aceleração vertical;

Lé a força horizontal no flange da roda;

$\mathrm{V}$ é a força vertical na pista roda.

Com base em valores de acelerações limite, comparadas à aceleração da gravidade, são definidos pontos de vibrações excessivas. É importante conhecer também as técnicas utilizadas na manutenção da via permanente e nas inspeções geométricas, onde são empregados carros controle, que realizam medições in situ e geram relatórios de exceções, nos quais as características como bitola, alinhamento, nivelamento longitudinal, torção (twist), superelevação e empeno (warp) aparecem como problemas. Essencialmente os parâmetros analisados são confrontados com os limites recomendados por normas.

Por outro lado, nas inspeções dinâmicas são empregados veículos especiais para medir acelerações, que atingem um alto nível de sofisticação. Além disso, as operações destes veículos inerciais devem ser programadas para evitar interferência com os trens regulares ou com outras tarefas de manutenção. Outras abordagens estão focadas no desenvolvimento de sistemas inerciais anexando acelerômetros ao truque de veículos ferroviários em serviço, porém, neste caso, há uma dificuldade do processamento de dados para obter resultados relevantes. Fundamentalmente, os parâmetros avaliados são as acelerações 
horizontais e verticais.

\section{Metodologia}

\subsection{Programa de Investigações}

O presente trabalho consiste em comparar os levantamentos geométricos obtidos com os equipamentos utilizados pelas operadoras, com os levantamentos obtidos por meio de acelerômetro portátil. Para tal, serão realizadas as coletas e separação dos relatórios de inspeção de geometria, geralmente compostos por gráficos de parâmetros geométricos versus localização, e planilha de exceções do trecho que se quer investigar. Nesses mesmos trechos, mantendo a quilometragem e sentido, é procedida a inspeção dinâmica.

Para a viabilização e verificação do trabalho foram realizados contatos com as operadoras MRS Logística e Supervia, que se prontificaram a disponibilizar os relatórios das inspeções geométricas realizadas pela empresa e permitiram as inspeções de dinâmica nos trechos.

Para este estudo, a escolha dos trechos monitorados baseou-se na relevância operacional das linhas, na existência de curvas com raios variados nos trechos específicos e nas tangentes bem definidas. Os critérios foram escolher trechos representativos em tangentes e curvas que possuíam defeitos registrados na inspeção dinâmica e não possuíam registros na inspeção de geometria, objetivando entender por que existem descarrilhamentos em locais onde não aparece defeitos de geometria, mas com uma interação veículo/via inadequada.

O procedimento de investigação de geometria e de dinâmica, proposto neste capítulo, foi aplicado a seis linhas ferroviárias de transporte de cargas e de passageiros, para demonstrar que as inspeções por vibração podem ser auxiliares às geométricas. No âmbito do transporte de carga analisaram-se dois trechos da malha ferroviária que interliga os Estados de Minas Gerais, São Paulo e Rio de Janeiro, da operadora MRS Logística S.A, que possui uma extensão total de aproximadamente 1.643 km. Os trechos analisados foram os de Miguel Burnier (do km 479 ao km 500; extensão inspecionada de 21 km) e F. Bernardino (do km 279 ao km 294; extensão inspecionada de 15 km).

No caso do transporte de passageiros, investigaram-se dois trechos sob administração da companhia de trens urbanos SuperVia, na região metropolitana do Rio de Janeiro, cuja malha possui extensão de $270 \mathrm{~km}$, dividida em cinco ramais, três extensões e 104 estações. Os trechos analisados foram os dos ramais de Japeri e Santa Cruz, em ambos os sentidos, totalizando quatro linhas, com extensões de $21 \mathrm{~km}$ e $32 \mathrm{~km}$, respectivamente. A Tabela 2 sumariza os trechos que foram utilizados neste trabalho.

Tabela 2. Informações sobre os trechos inspecionados.

\begin{tabular}{|c|c|c|c|c|c|c|c|c|}
\hline \multirow{2}{*}{ Ferrovia } & \multirow{2}{*}{ Descrição } & \multicolumn{2}{|c|}{ Trecho } & \multirow{2}{*}{ Carro-controle } & \multirow{2}{*}{$\begin{array}{c}\text { FRA* }^{*} \\
\text { Classe da Via }\end{array}$} & \multirow{2}{*}{ Total (Km) } & \multirow{2}{*}{$\begin{array}{c}\text { Data } \\
\text { Insp. Geo }\end{array}$} & \multirow{2}{*}{$\begin{array}{c}\text { Data } \\
\text { Insp.Din }\end{array}$} \\
\hline & & Início & Fim & & & & & \\
\hline MRS Logística & Burnier & $\mathrm{Km} 479$ & $\mathrm{Km} 500$ & TrackSTAR & 3 & 21 & $05 / 01 / 2016$ & $15 / 12 / 2017$ \\
\hline MRS Logística & Bernardino & $\mathrm{Km} 279$ & $\mathrm{Km} 300$ & TrackSTAR & 3 & 21 & 09/01/2018 & $29 / 01 / 2018$ \\
\hline Supervia & Japeri L1 & $\mathrm{Km} 23$ & Km61 & EM-80 ENSCO & 3 & 38 & $12 / 02 / 2019$ & $13 / 03 / 2019$ \\
\hline Supervia & Japeri L2 & $\mathrm{Km} 23$ & Km61 & EM-80 ENSCO & 3 & 38 & $12 / 02 / 2019$ & $13 / 03 / 2019$ \\
\hline Supervia & S. Cruz L1 & $\mathrm{Km} 22$ & $\mathrm{Km} 54$ & EM-80 ENSCO & 3 & 32 & $12 / 02 / 2019$ & $14 / 03 / 2019$ \\
\hline Supervia & S. Cruz L2 & $\mathrm{Km} 22$ & $\mathrm{Km} 54$ & EM-80 ENSCO & 3 & 32 & $12 / 02 / 2019$ & $14 / 03 / 2019$ \\
\hline
\end{tabular}

Nota*: De acordo com a classificação proposta pela Federal Railroad Administration (FRA), as ferrovias são enquadradas na Classe 3, com velocidade de tráfego entre $41 \mathrm{~km} / \mathrm{h}$ e $60 \mathrm{~km} / \mathrm{h}$. Fonte: Autores (2021).

Nestas ferrovias obtiveram-se dados de inspeção de geometria realizadas com os carros-controle. Foram levantados diversos parâmetros, dentre os quais foram alvo deste estudo: bitola, alinhamento, nivelamento longitudinal, superelevação e 
empeno (warp). A escolha destes parâmetros deve-se ao fato de terem sido os principais defeitos inspecionados nas análises de instrumentação da via permanente nas ferrovias, possuindo a representatividade necessária.

\subsection{Inspeção de Geometria com Veículos tipo Carro-Controle}

\subsubsection{Inspeção por Trackstar em Ramal de Transporte de Carga: Trecho da MRS Logística S.A.}

A MRS Logística S.A., que opera transporte de cargas entre Minas Gerais, Rio de Janeiro e São Paulo, utiliza o equipamento rodoferroviário (carro controle) chamado TrackSTAR, Figura 2, para realizar as inspeções de geometria em sua malha ferroviária.

Figura 2. Carro-controle TrackSTAR da MRS Logística.

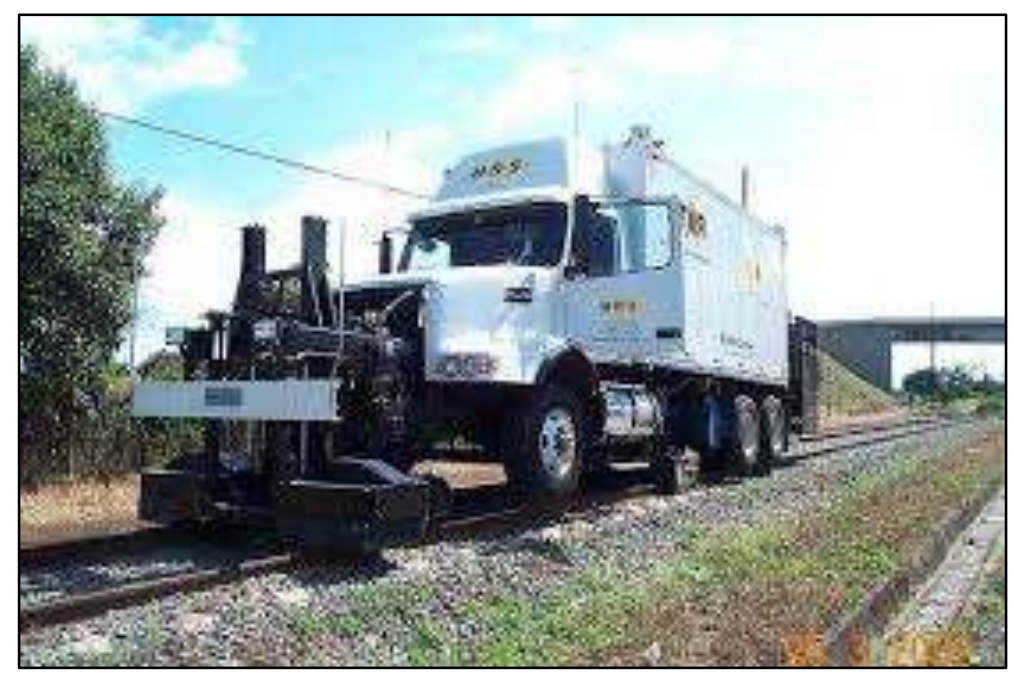

Fonte: Autores (2018).

O veículo tipo carro controle apresentado na Figura 2 é do modelo TrackStar (caminhão ferroviário), fabricado em 2001 pela Holland/Chicago e recebe upgrades com periodicidade de 2 em 2 anos. O carro controle é utilizado pela operadora ferroviária para a realização da inspeção geométrica da malha a fim de detectar a conformidade da via permanente. Os trechos escolhidos deste estudo foram o Ramal de Miguel Burnier e o trecho entre as estações Dias Tavares e Barbacena.

No momento da inspeção, se algum parâmetro geométrico coletado superar o respectivo limite previamente estabelecido, uma exceção desse parâmetro é registrada na lista de exceções que consolida o número da exceção, o maior valor coletado (no intervalo em que ocorre a exceção), a localização, a via, o tipo de exceção, entre outras informações.

\subsubsection{Inspeção de geometria do EM80 em Ramal de Transporte de Passageiros: Supervia}

O mesmo procedimento foi realizado com a empresa Supervia, que opera transporte de passageiros na região metropolitana do Rio de Janeiro. A Supervia utiliza um veículo ferroviário (carro controle) chamado EM80 que também fornece relatórios gráficos de geometria e lista de exceções.

A Figura 3 ilustra o veículo de carro-controle EM 80 usado nas vias da Supervia. 
Figura 3. Carro-controle EM-80 da Supervia.

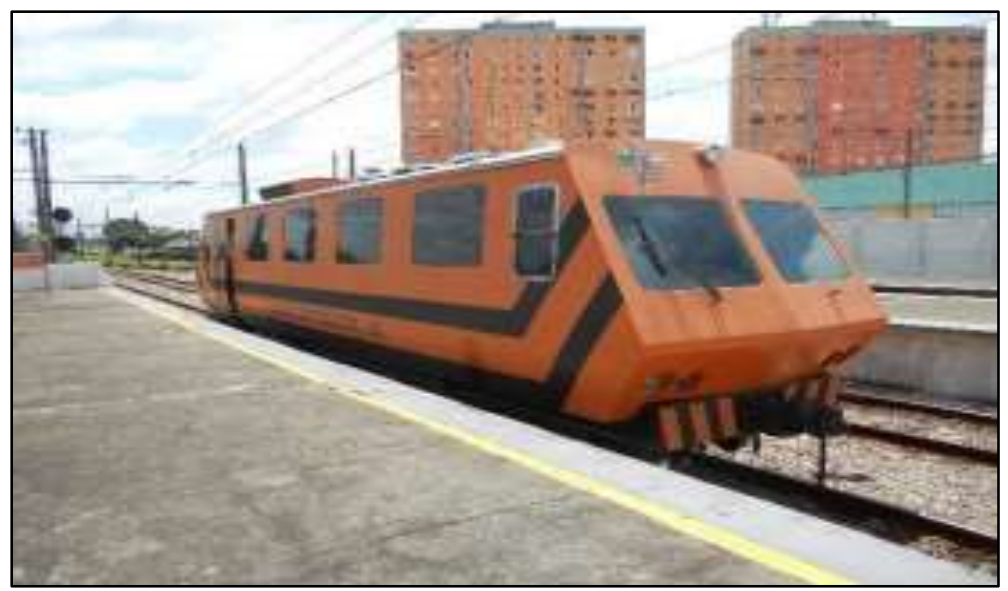

Fonte: Autores (2018).

O veículo da Figura 3 é do tipo carro-controle EM80 Plasser da década de 90 e recebe upgrades de 2 em 2 anos. Assim, como o carro-controle usado pela MRS, ambos veículos são usados na pesquisa pois são capazes realizar inspeções de geometria com altíssima precisão.

\subsection{Inspeção Dinâmica com Acelerômetros}

A inspeção dinâmica na via foi realizada utilizando um pacote inercial portátil (Figura 4), equipamento fabricado pela Kaneko Japan modelo KS8861A (handy vehicle jolt gauge accelerometer), que apresenta os valores de aceleração longitudinal e transversal, localização GPS (latitude e longitude) da exceção, quando ocorrer, além de possuir comunicação bluetooth com um tablet onde o inspetor vai incluindo observações relativas ao trecho (ex.: passagens em nível, pontes, túneis e etc).

Figura 4. Conjunto acelerômetro e tablet utilizados nas inspeções.
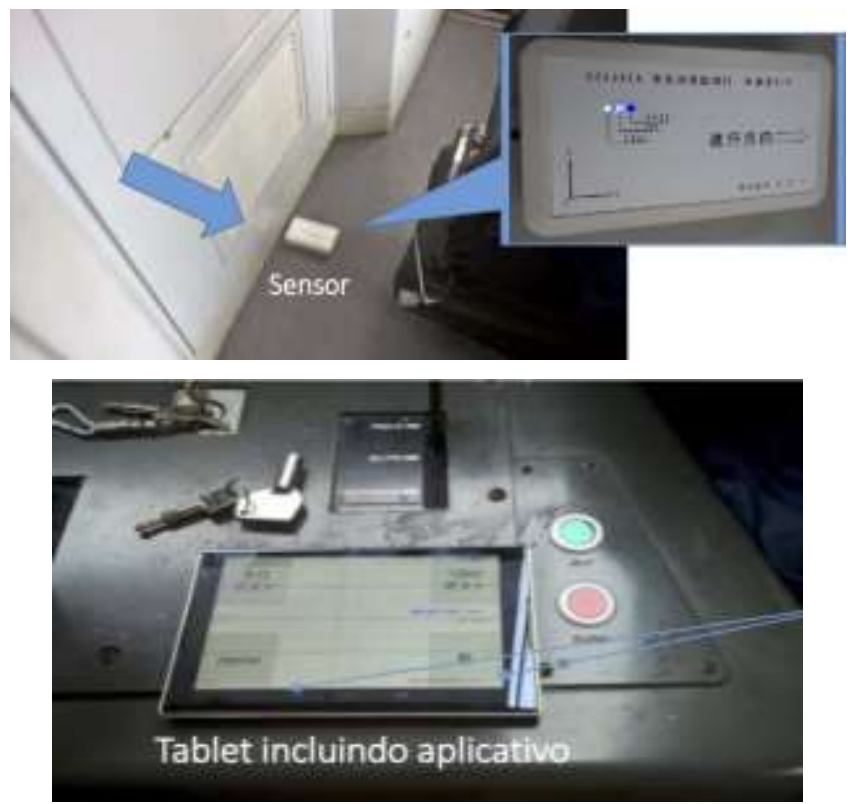

Fonte: Autores (2018).

O referido equipamento foi posicionado na cabine, no eixo de simetria das locomotivas e de TUEs (Trem Unidade Elétrica) para obtenção das acelerações horizontais e verticais medidas na circulação comercial dos trens. Como o mais 
importante é verificar os locais na via permanente onde as acelerações indesejadas acontecem, a locação do equipamento em outro ponto do material rodante não refletiria em resultados diferentes de inspeção da via. Por razões de segurança e facilidade de acesso ao equipamento, optou-se por colocá-lo na cabine por saber que os resultados de localização da não conformidade (latitude e longitude) não seriam alteradas ao longo do percurso.

Na avaliação dos dados, valores de acelerações horizontais e verticais maiores ou iguais a $10 \%$ e $15 \%$ da aceleração da gravidade, respectivamente, foram consideradas como exceções, conforme recomendação do fabricante.

Os quantitativos de exceções geométricas e acelerometria encontrados por linha investigada foram computados para análise. Cabe salientar que cada linha teve suas inspeções de geometria e dinâmica realizadas exatamente no mesmo trecho e sentido, sendo a inspeção de dinâmica no trem tipo que realiza a operação normal.

\section{Resultados e Discussão}

Os resultados foram obtidos a partir dos levantamentos de campo, realizados em seis linhas ferroviárias: M. Burnier, F. Bernardino, Japeri L1, Japeri L2, Santa Cruz L1 e Santa Cruz L2. Na análise dos resultados foi observado ainda a evidência de trechos onde existem muitas variações concomitantes dos parâmetros geométricos, sem que os limites de exceções fossem atingidos individualmente.

Os quantitativos de exceções geométricas e de acelerações encontrados para cada tipo de defeito, por linha investigada, foram computados para análise. A Figura 5 apresenta um exemplo onde pode-se observar que o L align (alinhamento esquerdo) e o R align (alinhamento direito) apresentavam variações próximas ao km 52,870, mas ainda dentro dos limites, não gerando exceção de geometria no trecho entre os $\mathrm{km} 52,800$ e 52,900.

Figura 5. Inspeção com carro controle entre o km 52,800 e km 52,900 na linha do Ramal de Santa Cruz.

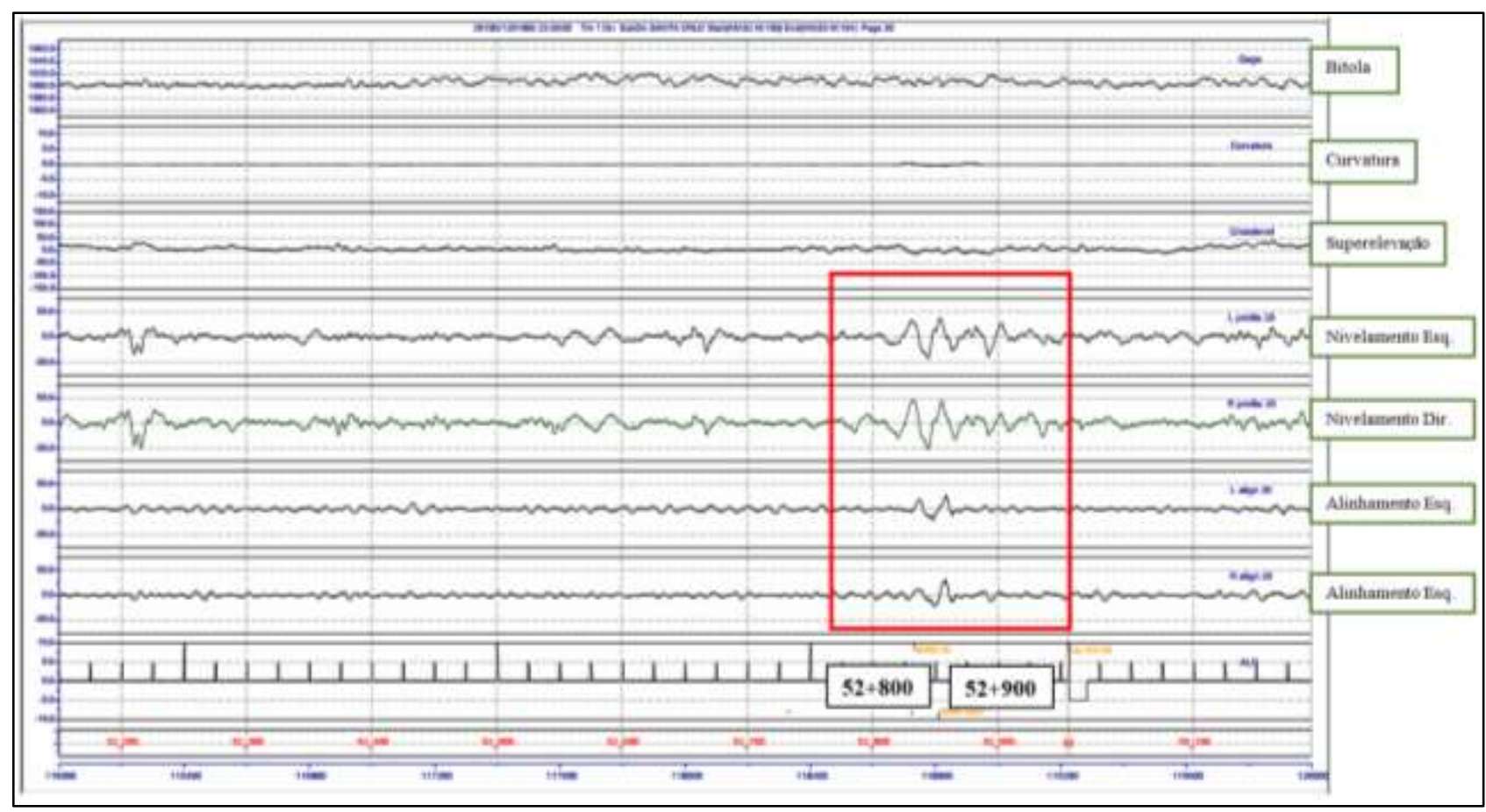

Fonte: Autores (2021).

Em contrapartida, quando realizada a inspeção dinâmica, verificou-se que entre o km 52,800 e o km 52,900 (Tabela 3) a inspeção dinâmica apontou acelerações não conformes gerando exceções de dinâmica. Pôde-se verificar que este trecho 
corresponde a uma região em que está instalada uma ponte na Linha 1 de S. Cruz, lugar que à luz da geometria pura e simples não entraria no plano de manutenção, mas ao realizarmos a inspeção dinâmica verificamos ser um ponto a ser tratado.

Tabela 3. Não conformidade dinâmica próximo ao km 52,870.

\begin{tabular}{ccccccccc}
\hline \multirow{2}{*}{ No. } & \multirow{2}{*}{ Km } & \multicolumn{3}{c}{ Vertical } & \multicolumn{3}{c}{ Horizontal } & \multirow{2}{*}{ Latitude Longitude } \\
\cline { 3 - 8 } & & max & Up & Down & max & Left & Right & $-22,9176-43,6592$ \\
$\mathbf{1}$ & 51,950 & $0,11 \mathrm{G}$ & 0,06 & $-0,05$ & $0,31 \mathrm{G}$ & 0,12 & $-0,19$ & $-22,9175-43,6594$ \\
$\mathbf{2}$ & 51,970 & $0,13 \mathrm{G}$ & 0,06 & $-0,07$ & $0,32 \mathrm{G}$ & 0,14 & $-0,18$ & $-22,9165-43,6673$ \\
$\mathbf{3}$ & 51,790 & $0,21 \mathrm{G}$ & 0,11 & $-0,11$ & $0,33 \mathrm{G}$ & 0,17 & $-0,16$ & $-22,9164-43,6683$ \\
$\mathbf{4}$ & 51,870 & $0,43 \mathrm{G}$ & 0,22 & $-0,21$ & $0,16 \mathrm{G}$ & 0,03 & $-0,13$ & $-22,9164-43,6695$ \\
$\mathbf{5}$ & 51,890 & $0,47 \mathrm{G}$ & 0,31 & $-0,16$ & $0,08 \mathrm{G}$ & 0,03 & $-0,05$ & \\
\hline
\end{tabular}

Fonte: Autores (2021).

Da mesma forma, a metodologia foi aplicada em todos os trechos experimentados no estudo. Os quantitativos de exceções de bitola (B), alinhamento (A), nivelamento longitudinal (NL), superelevação (S), empeno (E), aceleração vertical (Av) e aceleração horizontal (Ah) das ferrovias inspecionadas são apresentados na Tabela 4. Os totais de não conformidades por linha e tipo de inspeção são resumidos na Figura 6.

Tabela 4. Exceções apresentadas pelas ferrovias.

\begin{tabular}{|c|c|c|c|c|c|c|c|c|}
\hline \multirow[b]{2}{*}{ Linha } & \multirow[b]{2}{*}{ Tipo } & \multicolumn{5}{|c|}{ Geometria } & \multicolumn{2}{|c|}{ Dinâmica } \\
\hline & & Bitola & Alinhamento & $\begin{array}{l}\text { Nivelamento } \\
\text { longitudinal }\end{array}$ & Superelevação & Empeno & Vmax & Hmax \\
\hline Burnier & Carga & 6 & 0 & 0 & 0 & 0 & 11 & 6 \\
\hline F. Bernardino & Carga & 0 & 0 & 0 & 1 & 0 & 25 & 12 \\
\hline Japeri L1 & Passageiros & 4 & 0 & 0 & 0 & 0 & 0 & 1 \\
\hline Japeri L2 & Passageiros & 3 & 0 & 0 & 0 & 0 & 6 & 2 \\
\hline S. Cruz L1 & Passageiros & 1 & 1 & 0 & 15 & 11 & 62 & 23 \\
\hline S. Cruz L2 & Passageiros & 1 & 0 & 2 & 23 & 12 & 22 & 56 \\
\hline \multicolumn{2}{|c|}{ Total } & 15 & 1 & 2 & 39 & 23 & 126 & 98 \\
\hline
\end{tabular}

Fonte: Autores (2021).

Figura 6. Não conformidades por linha, dinâmica e geometria.

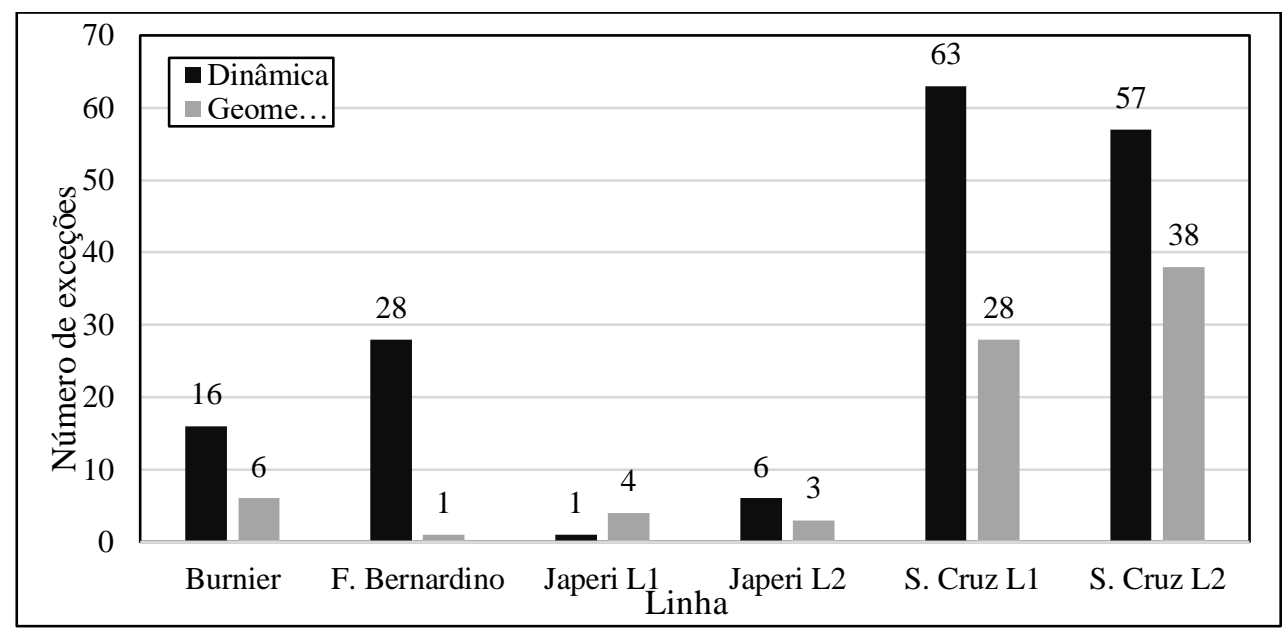

Fonte: Autores (2021).

Quando comparadas as linhas da ferrovia de carga e de passageiro na Tabela 4 e na Figura 6, percebe-se que a via permanente se encontra em melhor estado no primeiro grupo. As linhas do ramal Santa Cruz foram as que apresentaram os piores 
indicadores da via permanente, seguidas das linhas F. Bernardino, Burnier e Japeri. Em alguns pontos da investigação ocorreu mais de uma não conformidade, como, por exemplo, acelerações horizontais e verticais fora dos limites ou superelevação e empeno simultaneamente.

Partindo para a análise dos números totais de exceções geométricas (NEG) e exceções dinâmicas (NED), estes foram primeiramente subdivididos por linha investigada. Nesse contexto, após a análise de todos os ramais ferroviários da pesquisa, foi possível identificar trechos com exceções coincidentes (NEC) (ou seja, que apresentaram exceções na inspeção geométrica e na dinâmica no mesmo trecho), o total de exceções (NTE), além de porcentagens coincidentes (PC), de exceções geométricas (PEG) e de exceções dinâmicas (PED) por linha ferroviária. Tais resultados são apresentados na Tabela 5.

Tabela 5. Número de Exceções Coincidentes (NEC), Número de Exceções Geométricas (NEG), Número de Exceções de Dinâmica (NED) e Número Totais de Exceções (NTE) e as respectivas porcentagens: Coincidentes (PC), de Exceções Geométricas (PEG) e de Exceções de Dinâmica (PED) para os trechos investigados no estudo.

\begin{tabular}{cccccccc}
\hline Linha & NEC (un.) & NEG (un.) & NED (un.) & NTE (un.) & PC (\%) & PEG (\%) & PED (\%) \\
\hline Burnier & 2 & 6 & 16 & 22 & 9,09 & 27,27 & 72,73 \\
F. Bernardino & 0 & 1 & 28 & 29 & 0,00 & 3,45 & 96,55 \\
Japeri L1 & 0 & 4 & 1 & 5 & 0,00 & 80,00 & 20,00 \\
Japeri L2 & 1 & 3 & 6 & 9 & 11,11 & 33,33 & 66,67 \\
S. Cruz L1 & 6 & 28 & 63 & 91 & 6,59 & 30,77 & 69,23 \\
S. Cruz L2 & 11 & 38 & 57 & 95 & 11,58 & 40,00 & 60,00 \\
Total & 20 & 80 & 171 & 251 & 7,97 & 31,87 & 68,13 \\
\hline
\end{tabular}

Fonte: Autores (2021).

Nesse contexto, de acordo com os dados da Tabela 5, foi possível identificar, para cada linha, que:

- A linha Burnier apresentou 16 exceções dinâmicas (NED), sendo que em um ponto ocorreu não conformidade de aceleração vertical e horizontal simultaneamente. Na inspeção de geometria foram identificados seis pontos (NEG = 6) com exceção de bitola. Não houve ocorrência de mais de uma não conformidade de geometria em um mesmo ponto. A referida linha apresentou dois pontos com exceções de dinâmica e geometria coincidentes $(\mathrm{NEC}=2)$.

- A linha Francisco Bernardino apresentou 28 exceções dinâmicas (NED), sendo que em nove pontos ocorreu não conformidade de aceleração vertical e horizontal simultaneamente. Na inspeção de geometria foi identificado um ponto com exceção de superelevação $(\mathrm{NEG}=1)$. Não houve ocorrência de mais de uma não conformidade de geometria em um mesmo ponto. A referida linha não apresentou pontos com exceção de dinâmica e geometria coincidentes $(\mathrm{NEC}=0)$.

- A linha Japeri 1 apresentou uma exceção de aceleração horizontal (NED = 1). Na inspeção de geometria foram identificados quatro pontos com exceção de bitola $(\mathrm{NEG}=4)$. Não houve ocorrência de mais de uma não conformidade de geometria em um mesmo ponto. A referida linha não apresentou pontos com exceções de dinâmica e geometria coincidentes $(\mathrm{NEC}=0)$.

- A linha Japeri 2 apresentou seis exceções de acelerações (NED =6), sendo que em dois pontos ocorreram não conformidades de acelerações e horizontais simultaneamente. Na inspeção de geometria foram identificados três pontos com exceção de bitola $(\mathrm{NEG}=3)$. Não houve pontos com ocorrência de mais de um defeito de geometria. A referida linha apresentou um ponto com exceção de dinâmica e geometria coincidentes $(\mathrm{NEC}=1)$.

- A linha Santa Cruz 1 apresentou 63 exceções de acelerações (NED = 63), sendo que em 22 pontos ocorreram não conformidades de acelerações e horizontais simultaneamente. $\mathrm{Na}$ inspeção de geometria foram identificados 28 pontos com exceções de superelevação, empeno, bitola e alinhamento (NEG =28), com ocorrência individual ou simultânea. A referida linha apresentou seis pontos com exceção de dinâmica e geometria coincidentes $(\mathrm{NEC}=6)$. No gráfico o número de exceções é maior 
que o número de pontos pois em um único ponto pode acontecer 2 ou mais exceções.

- A linha Santa Cruz 2 apresentou 58 exceções de acelerações (NED = 58), sendo que em 21 pontos ocorreram não conformidades de acelerações e horizontais simultaneamente. Na inspeção de geometria foram identificados 38 pontos com exceções de superelevação, empeno, bitola e nivelamento longitudinal ( $\mathrm{NEG}=38$ ), com ocorrência individual ou simultânea. A referida linha apresentou 11 pontos com exceção de dinâmica e geometria coincidente (NEC $=11$ ). No gráfico o número de exceções é maior que o número de pontos pois em um único ponto pode acontecer 2 ou mais exceções.

De uma forma geral pode-se afirmar, que a maior parte das exceções registradas pelos sistemas de medição foram dinâmicas, representando 68,13\% do total (Total do PED, NED = 171). Em quatro das seis linhas, houve casos em que os pontos avaliados coincidiram, representando 7,97\% do total (Total do PC, NEC $=20$ ). Estes pontos são de alta criticidade e necessitam de diagnóstico e manutenção prioritária.

Posteriormente, como forma de verificar a correspondência entre as exceções de geometria e de dinâmica já apresentadas, obteve-se a matriz de correlação dos parâmetros, cujo resultado é mostrado na Tabela 6 e comparado com a classificação da intensidade da correlação (Tabela 7).

Tabela 6. Matriz de correlação das exceções.

\begin{tabular}{|c|c|c|c|c|c|c|c|}
\hline & B & $\mathbf{A}$ & NL & $\mathbf{S}$ & $\mathbf{E}$ & $\mathbf{A v}$ & Ah \\
\hline B & 1,000 & & & & & & \\
\hline A & $-0,325$ & 1,000 & & & & & \\
\hline NL & $-0,325$ & $-0,200$ & 1,000 & & & & \\
\hline$S$ & $-0,526$ & 0,416 & 0,807 & 1,000 & & & \\
\hline $\mathrm{E}$ & $-0,514$ & 0,590 & 0,673 & 0,979 & 1,000 & & \\
\hline Av & $-0,586$ & 0,905 & 0,022 & 0,578 & 0,701 & 1,000 & \\
\hline $\mathrm{Ah}$ & $-0,530$ & 0,148 & 0,922 & 0,951 & 0,872 & 0,404 & 1,000 \\
\hline
\end{tabular}

Fonte: Autores (2021).

Tabela 7. Classificação da intensidade da correlação.

\begin{tabular}{cl}
\hline Valor & \multicolumn{1}{c}{ Intensidade da Correlação } \\
\hline 0,9 & Para mais ou menos indica uma correlação muito forte \\
0,7 a 0,9 & Positivo ou negativo indica uma correlação forte \\
0,5 a 0,7 & Positivo ou negativo indica uma correlação moderada \\
0,3 a 0,5 & Positivo ou negativo indica uma correlação fraca \\
0,0 a 0,3 & Positivo ou negativo indica uma correlação desprezível \\
\hline
\end{tabular}

Fonte: Callegari-Jacques (2003).

De acordo com a Tabela 6 e a Tabela 7, verifica-se que, para o conjunto de dados analisado, houve uma correlação forte e muito forte, respectivamente, (conforme a classificação de Callegari-Jacques (2003)) das superelevações (S) com os nivelamentos longitudinais (NL) $(0,807)$ e o empeno (E) $(0,979)$; muito forte entre as acelerações verticais (Av) com os alinhamentos (A) $(0,905)$ e forte da Av com o empeno (E) $(0,701)$; muito forte entre das acelerações horizontais (Ah) com os nivelamentos longitudinais (NL) $(0,922)$ e muito forte entre a Ah e empeno (E) $(0,951)$. Ou seja, dos parâmetros geométricos, apenas a bitola não apresentou correspondência com as acelerações (- 0,586 com Av e -0,530 com Ah).

Com os resultados obtidos, verificou-se que as inspeções dinâmicas apresentaram um número maior de exceções quando comparadas às inspeções de geometria. Quando analisados os gráficos de geometria, nas regiões onde se localizavam apenas exceções dinâmicas, percebeu-se que geralmente havia variação nos parâmetros de geometria, porém tais variações não atingiam os limites definidos como exceções geométricas. Observou-se ainda que independentemente do tipo da linha, se de passageiro ou de carga esta situação se repete, confirmando-se assim a boa correlação e complementariedade das medições geométricas e 
dinâmicas, e a importância do procedimento sugerido neste estudo. Ou seja, é razoável inferir que só com a inspeção de geometria algumas não-conformidades potencialmente associadas a descarrilamento podem vir a ser negligenciadas.

\section{Considerações Finais}

A ocorrência de descarrilamentos em sistemas ferroviários brasileiros ainda é um problema recorrente. As falhas na superestrutura são frequentemente associadas a este fenômeno. Como método de identificação de potenciais pontos de descarrilamento comumente, utilizam-se as inspeções de geometria. No entanto, diante da evolução dos estudos em ferrovias e experiências internacionais, a adoção de critérios que levem em consideração a dinâmica da ferrovia são sugeridos como um caminho para minorar a ocorrência de acidentes nas ferrovias. Este artigo apresentou uma metodologia para identificação de trechos com potencial de descarrilamento com base na inspeção geométrica e na inspeção dinâmica, sendo esta por meio de um pacote inercial de acelerômetros.

O dispositivo inercial apresentado no artigo foi capaz de identificar pontos críticos das malhas ferroviárias de carga e de passageiros, que, em sua maioria, não apresentavam problemas quanto às inspeções geométricas nestes locais. Além disso, foram detectados trechos com forte potencial de descarrilamento, pois possuíam exceções de geometria e dinâmica simultaneamente, indicando um defeito na via permanente. Deve-se salientar que existem outras metodologias de investigação dinâmica de uma ferrovia, mas que o custo envolvido com carros exclusivos para este fim, ou ainda a instrumentação dos truques, é menos operacional e mais cara que a utilização de acelerômetros digitais portáteis, que geram os relatórios instantaneamente. Portanto, a utilização dos sistemas de medição de geometria aliados aos acelerômetros, podem auxiliar os mantenedores ferroviários a elaborar planos de manutenção mais assertivos.

Por fim, deve-se salientar que a inspeção dinâmica aqui apresentada não substitui as inspeções de geometria, mas sim complementam com dados importantes para uma melhor priorização de quais exceções devem receber manutenções corretivas primeiro. Este trabalho propõe assim complementar a inspeção de geometria com a inspeção dinâmica utilizando pacotes inerciais portáteis buscando uma melhor e mais efetiva manutenção de via permanente com vistas a diminuir descarrilamentos.

\section{Agradecimentos}

Os autores agradecem à ViaMOB Engenharia, à SuperVia, à MRS Logística S.A. e ao IME - Instituto Militar de Engenharia.

\section{Referências}

Arivazhagan, S., Newlin Shebiah, R., Salsome Magdalene, J., \& Sushmitha, G. (2015). Railway track derailment inspection system using segmentation-based fractal texture analysis. ICTACT Journal on Image and Video Processing, 06(01), 1060-1065. doi:10.21917/ijivp.2015.0155

Boogaard, M. A., Li, Z., \& Dollevoet, R. P. B. J. (2018). In situ measurements of the crossing vibrations of a railway turnout. Measurement, 125, 313-324. 10.1016/j.measurement.2018.04.094

Brina, H. L. (1983). Estradas de Ferro. Livros Tecnicos e Cientificos Ed.

Callegari-Jacques, S. M. (2003). Bioestatística: Princípios e Aplicações. Artmed.

Campos, P. C. O. (2019). Avaliação do efeito da variação da umidade no comportamento mecanístico de um trecho da Estrada de Ferro Carajás (Dissertação de Mestrado). Instituto Militar de Engenharia, Programa de Pós-Graduação em Engenharia de Transportes, Rio de Janeiro.

Cherchas, D. B. (1981). Determination of railway wheel climb probability based on the derailment coefficient. Journal of the Franklin Institute, 312(1), 31-40. 10.1016/0016-0032(81)90070-3

DIN EN 12229 (2009). Railway applications - Ride comfort for passengers - measurement and evaluation. British Standards Institution.

Indian Railways Institute of Civil Engineering. (2014). The investigation of derailments. IRICEN.

Iwnick, H. (2006). Handbook of Railway Vehicle Dynamics. CRC Press. 10.1201/9781420004892 
Research, Society and Development, v. 10, n. 6, e3910615369, 2021

(CC BY 4.0) | ISSN 2525-3409 | DOI: http://dx.doi.org/10.33448/rsd-v10i6.15369

Kasraei, A., \& Zakeri, J. A. (2020). Effective time interval for railway track geometry inspection. Archives of Transport, 53(1), 53-65. $10.5604 / 01.3001 .0014 .1744$

Liu, X., Barkan, C. P. L., \& Saat, M. R. (2011). Analysis of Derailments by Accident Cause. Transportation Research Record: Journal of the Transportation Research Board, 2261(1), 178-185. 10.3141/2261-21

Liu, Y., \& Magel, E. (2012). Understanding Wheel-Climb Derailments. In Railway Track and Structures (12a ed.), 103, 37-41. Simmons-Boardman Publishing Corporation. https://trid.trb.org/view/842625

Lopes, \& Ciriaco, F. (2017). Análise e prevenção de descarrilamentos. Fundamentos de Engenharia Ferroviária, Instituto Militar de Engenharia.

Morales-Ivorra, S., Real, J. I., Hernández, C., \& Montalbán, L. (2016). Derailment risk and dynamics of railway vehicles in curved tracks: Analysis of the effect of failed fasteners. Journal of Modern Transportation, 24(1), 38-47. 10.1007/s40534-015-0093-z

Ngamkhanong, C., Kaewunruen, S., \& Costa, B. (2018). State-of-the-Art Review of Railway Track Resilience Monitoring. Infrastructures, 3(1), 3. $10.3390 /$ infrastructures3010003

Pastori, A. (2007). As PPPs como ferramenta para viabilizar projetos de infra-estrutura ferroviária um estudo para reativação do trem de passageiros sobre trilhos. Revista do BNDES, 14(28), 93-100.

Ratton Neto, H. X. (1985). Padrões para Manutenção da Via Permanente (Dissertação de Mestrado). Instituto Militar de Engenharia, Programa de PósGraduação em Engenharia de Transportes.

Real, J. I., Montalbán, L., Real, T., \& Puig, V. (2012). Development of a system to obtain vertical track geometry measuring axle-box accelerations from in service trains. Journal of Vibroengineering, 14(2), 813-826. https://www.jvejournals.com/article/10639.

Salvador, P., Naranjo, V., Insa, R., \& Teixeira, P. (2016). Axlebox accelerations: Their acquisition and time-frequency characterisation for railway track monitoring purposes. Measurement, 82, 301-312. doi:10.1016/j.measurement.2016.01.012

Santos, G. F. M. dos, \& Barbosa, R. S. (2016). Safety analysis of a railway car under the periodic excitation from the track. Cogent Engineering, 3(1), 1263027. doi:10.1080/23311916.2016.1263027

Sato, Y., \& Umehara, T. (1987). Track maintenance engineering. JRCEA.

Selig, E. T., \& Waters, J. M. (1994). Track Geotechnology and Substructure Management. Thomas Telford Publishing. 10.1680/tgasm.20139

Valinho, M. N., Cescon, J. T. A. M., Simoni, A. P. R., \& Zambrotti, L. de L. P. (2020). Socioeconomic impacts with the implementation of the railroad EF-118 RJ/ES: comparison of cargo logistics at the Port of Açu. Research, Society and Development, 9(9), e995998389. doi:10.33448/rsd-v9i9.8389

Vlakhova, A. V. (2015). Risk assessment of flange climb derailment of a rail vehicle. Mechanics of Solids, 50(1), 19-32. 10.3103/S0025654415010033

Wang, W., \& Li, G. (2012). Development of high-speed railway vehicle derailment simulation - Part II: Exploring the derailment mechanism. Engineering Failure Analysis, 24, 93-111. 10.1016/j.engfailanal.2012.02.001

Zeng, J., \& Wu, P. (2008). Study on the wheel/rail interaction and derailment safety. Wear, 265(9-10), 1452-1459. 10.1016/j.wear.2008.01.031 